PROCEEDINGS OF THE

AMERICAN MATHEMATICAL SOCIETY

Volume 126, Number 6, June 1998, Pages 1685-1695

S 0002-9939(98)04310-X

\title{
RENORMALIZED OSCILLATION THEORY FOR DIRAC OPERATORS
}

\author{
GERALD TESCHL
}

(Communicated by Palle E. T. Jorgensen)

\begin{abstract}
Oscillation theory for one-dimensional Dirac operators with separated boundary conditions is investigated. Our main theorem reads: If $\lambda_{0,1} \in \mathbb{R}$ and if $u, v$ solve the Dirac equation $H u=\lambda_{0} u, H v=\lambda_{1} v$ (in the weak sense) and respectively satisfy the boundary condition on the left/right, then the dimension of the spectral projection $P_{\left(\lambda_{0}, \lambda_{1}\right)}(H)$ equals the number of zeros of the Wronskian of $u$ and $v$. As an application we establish finiteness of the number of eigenvalues in essential spectral gaps of perturbed periodic Dirac operators.
\end{abstract}

\section{INTRODUCTION}

In [16] Sturm originated oscillation theory for second-order differential equations one hundred and fifty years ago. Since then numerous extensions have been made (see, e.g., [2],[11],[14],[17], and the references therein). In [24] Weidmann extended results for Sturm-Liouville operators from Hartman [5], [6], Hartman and Putnam [7], and himself [23] to the case of Dirac operators. In particular, he proves Sturm-type comparison theorems and applies the results to investigate the essential spectrum of Dirac operators. With the present paper we want to complement [24] in the sense that we will use oscillation theory to investigate the discrete spectrum.

Using standard oscillation theory would mean to count zeros of components of solutions of the Dirac equation. Unfortunately this approach soon leads into severe troubles:

(i). Components of solutions might vanish identically on some intervals.

(ii). Zeros of components of solutions are not monotone with respect to the spectral parameter. Hence solutions can pick up or lose zeros as the spectral parameter increases, which, in general, destroys the connection between zeros and number of eigenvalues (cf. Remark 3.3).

The natural remedy is to look at zeros of the Wronskian instead, that is, use a renormalized version of oscillation theory developed in [4] for the case of SturmLiouville operators (see [18] in the case of Jacobi operators). In addition, this approach avoids technical difficulties arising from the fact that Dirac operators, in contradistinction to Sturm-Liouville operators, are not bounded from below.

Received by the editors November 7, 1996.

1991 Mathematics Subject Classification. Primary 34C10, 39L40; Secondary 34B24, 34L15.

Key words and phrases. Oscillation theory, Dirac operators, spectral theory.

(C) 1998 by the author 
To set the stage, let $I=(a, b) \subseteq \mathbb{R}$ (with $-\infty \leq a<b \leq \infty$ ) be an arbitrary interval and consider the Dirac differential expression

$$
\tau=\frac{1}{\mathrm{i}} \sigma_{2} \frac{d}{d x}+\phi(x) .
$$

Here

$$
\phi(x)=\phi_{\mathrm{el}}\left(m+(x) \mathbb{1}+\phi_{\mathrm{am}}(x) \sigma_{1}+\phi_{\mathrm{sc}}(x)\right) \sigma_{3},
$$

$\sigma_{1}, \sigma_{2}, \sigma_{3}$ denote the Pauli matrices

$$
\sigma_{1}=\left(\begin{array}{cc}
0 & 1 \\
1 & 0
\end{array}\right), \quad \sigma_{2}=\left(\begin{array}{cc}
0 & -\mathrm{i} \\
\mathrm{i} & 0
\end{array}\right), \quad \sigma_{3}=\left(\begin{array}{cc}
1 & 0 \\
0 & -1
\end{array}\right),
$$

and $m, \phi_{\mathrm{sc}}, \phi_{\mathrm{el}}$, and $\phi_{\mathrm{am}}$ are interpreted as mass, scalar potential, electrostatic potential, and anomalous magnetic moment, respectively (see [19], Chapter 4). As usual we require $m \in[0, \infty)$ and $\phi_{\mathrm{sc}}, \phi_{\mathrm{el}}, \phi_{\mathrm{am}} \in L_{l o c}^{1}(I, \mathbb{R})$ real-valued. We don't include a magnetic moment $\hat{\tau}=\tau+\sigma_{2} \phi_{\mathrm{mg}}(x)$, since it can be easily eliminated by a simple gauge transformation $\tau=U \hat{\tau} U^{-1}, U=\exp \left(\mathrm{i} \int{ }^{x} \phi_{\mathrm{mg}}(t) d t\right)$ (there is also a gauge transformation which gets rid of $\phi_{a m}$ or $\phi_{\mathrm{el}}$ (see [12], Section 7.1.1)). Explicitly we have

$$
\tau f=\left(\begin{array}{cc}
\phi_{11} & -\frac{d}{d x}+\phi_{12} \\
\frac{d}{d x}+\phi_{12} & \phi_{22}
\end{array}\right)\left(\begin{array}{c}
f_{1} \\
f_{2}
\end{array}\right)=\left(\begin{array}{c}
f_{2}^{\prime}+\phi_{12} f_{2}+\phi_{11} f_{1} \\
-f_{1}^{\prime}+\phi_{12} f_{1}+\phi_{22} f_{2}
\end{array}\right),
$$

$f \in A C_{l o c}\left(I, \mathbb{C}^{2}\right)$, where primes denote derivatives with respect to $x$ and $\phi_{11}=$ $\phi_{\mathrm{el}}+m+\phi_{\mathrm{sc}}, \phi_{12}=\phi_{21}=\phi_{\mathrm{am}}, \phi_{22}=\phi_{\mathrm{el}}-m-\phi_{\mathrm{sc}}$.

If $\tau$ is limit point at both $\pm \infty$, then $\tau$ gives rise to a unique self-adjoint operator $H$ when defined maximally (cf., e.g., [12], [22], [24]). Otherwise, we fix a boundary condition at each endpoint where $\tau$ is limit circle.

By $u_{ \pm}(z, x)$ we will denote (not identically vanishing) solutions of the differential equation $\tau u=z u, z \in \mathbb{C}$, which satisfy the following requirements (whenever such solutions exist).

(i). $u_{ \pm}(z,.) \in A C_{l o c}\left(I, \mathbb{C}^{2}\right)$ and $\tau u_{ \pm}(z)=z u_{ \pm}(z)$.

(ii). $u_{+}(z,$.$\left.) (resp. u_{-}(z,).\right)$ is square integrable near $b$ (resp. $a$ ) and fulfills the boundary condition of $H$ at $b$ (resp. $a$ ) if any (i.e., if $\tau$ is limit circle at $b$ (resp. a)).

Explicitly, $H$ is given by

$$
\begin{aligned}
H: \mathfrak{D}(H) & \rightarrow L^{2}\left(I, \mathbb{C}^{2}\right), \\
f & \mapsto \tau f,
\end{aligned}
$$

where

$$
\begin{aligned}
\mathfrak{D}(H)=\left\{f \in L^{2}\left(I, \mathbb{C}^{2}\right) \mid \begin{array}{l}
f \in A C_{\text {loc }}\left(I, \mathbb{C}^{2}\right), \tau f \in L^{2}\left(I, \mathbb{C}^{2}\right), \\
\left.W_{a}\left(u_{-}\left(\lambda_{0}\right), f\right)=W_{b}\left(u_{+}\left(\lambda_{0}\right), f\right)=0\right\}
\end{array}\right.
\end{aligned}
$$

with

$$
W_{x}(f, g)=f_{1}(x) g_{2}(x)-f_{2}(x) g_{1}(x),
$$

the usual Wronskian (we remark that the the limit $W_{a, b}(., .)=.\lim _{x \rightarrow a, b} W_{x}(., .$. exists for functions as in (1.6)). The resolvent of $H$ can be expressed in terms of $u_{ \pm}(z)$ as follows:

$$
(H-z)^{-1} f(x)=\int_{a}^{b} G(z, x, y) f(y) d y
$$


where

$$
G(z, x, y)=\frac{u_{ \pm}(z, x) \otimes u_{\mp}(z, y)}{W\left(u_{+}(z), u_{-}(z)\right)}, \quad \pm(x-y)>0 .
$$

Recall that $W_{x}\left(u_{+}(z), u_{-}(z)\right)$ is independent of $x$ (cf. (2.1)). In addition, we set $G(z, x, x)=\lim _{\varepsilon \rightarrow 0}(G(z, x+\varepsilon, x)+G(z, x-\varepsilon, x)) / 2$.

Denote by $H_{x,-}\left(\right.$ resp. $\left.H_{x,+}\right), x \in I$, self-adjoint operators associated with $\tau$ on $L^{2}\left((a, x), \mathbb{C}^{2}\right)$ (resp. $\left.L^{2}\left((x, b), \mathbb{C}^{2}\right)\right)$ obtained from $H$ by imposing the additional boundary condition $f_{1}(x)=0$. Then $H_{x,-} \oplus H_{x,+}$ is a rank one resolvent perturbation of $H$, and hence $\sigma_{\text {ess }}(H)=\sigma_{\text {ess }}\left(H_{x,-}\right) \cup \sigma_{\text {ess }}\left(H_{x,+}\right)$ (cf. [25], Korollar 6.2). Here $\sigma_{e s s}($.$) denotes the essential spectrum. If G_{x, \pm}(z, ., .$.$) denotes the resolvent$ kernel of $H_{x, \pm}$, we define the Weyl $m$-functions $m_{x, \pm}(z)$ (w.r.t. the base point $x$ ) by

$$
G_{x, \pm}(z, x, x)=\left(\begin{array}{cc}
0 & \pm \frac{1}{2} \\
\pm \frac{1}{2} & m_{x, \pm}(z)
\end{array}\right)
$$

The first resolvent identity shows that $m_{x, \pm}(z)$ are Herglotz functions (cf., e.g., $[20])$.

Lemma 1.1. The solutions $u_{ \pm}(z, x)$ exist for $z \in \mathbb{C} \backslash \sigma_{\text {ess }}\left(H_{x_{0}, \pm}\right)$. They can be assumed real analytic with respect to $z \in \mathbb{C} \backslash \sigma\left(H_{x_{0}, \pm}\right)$. In addition, we can include a finite number of isolated eigenvalues in the domain of holomorphy of $u_{ \pm}(z, x)$ by removing the corresponding poles.

Proof. If $U\left(z, x, x_{0}\right), z \in \mathbb{C}$, is a fundamental matrix solution for $\tau u=z u$ (i.e., $\left.U\left(z, x_{0}, x_{0}\right)=\mathbb{1}, x_{0} \in I\right)$ and $m_{x_{0}, \pm}(z)$ are the Weyl $m$-functions with respect to the base point $x_{0}$, then we can choose

$$
u_{ \pm}(z, x)=U\left(z, x, x_{0}\right)\left(\begin{array}{c}
1 \\
\pm m_{x_{0}, \pm}(z)
\end{array}\right) .
$$

By removing the corresponding poles of $m_{x_{0}, \pm}(z)$ we can include a finite number of isolated eigenvalues in the domain of holomorphy of $u_{ \pm}(z, x)$.

A finite end point is called regular if $\phi_{11}, \phi_{12}, \phi_{22}$ are integrable near this end point. In this case boundary values for all functions exist at this end point. In particular, $\tau$ is called regular if both end points $a, b$ are regular, that is, $a, b \in \mathbb{R}$ and $\phi_{11}, \phi_{12}, \phi_{22} \in L^{1}(I, \mathbb{R})$. In the regular case the resolvent of $H$ is HilbertSchmidt, and hence the spectrum is purely discrete (i.e., $\sigma_{\text {ess }}(H)=\emptyset$ ).

\section{WRONSKIANS}

In this section we want to investigate the Wronskian of two solutions $u, v$. A straightforward calculation gives

$$
W_{x}^{\prime}(u, v)=\left(\lambda_{1}-\lambda_{0}\right) u(x) v(x)
$$

if $\tau u=\lambda_{0} u$ and $\tau v=\lambda_{1} v$. Note that (in contradistinction to the Sturm-Liouville case) the Wronskian of two solutions can only have simple zeros (unless $\lambda_{0}=\lambda_{1}, u=$ $v$ or $u \equiv 0$ (resp. $v \equiv 0$ ) of course). Moreover, $W_{x}(u, v)=0$ if $u(x), v(x)$ are parallel and $W_{x}^{\prime}(u, v)=0$ if $u(x), v(x)$ are orthogonal. 
Clearly this implies

Lemma 2.1. Let $\tau u=\lambda_{0} u$ and $\tau v=\lambda_{1} v$ for some $\lambda_{1} \neq \lambda_{0}$. If $u, v \in L^{2}\left((c, d), \mathbb{C}^{2}\right)$ and $W_{c}(u, v)=W_{d}(u, v)$ for some $a \leq c<d \leq b$, then $u$, $v$ are orthogonal on $(c, d)$, that is, $\int_{c}^{d} u(t) v(t) d t=0$.

Proof. Integrating (2.1) we obtain $W_{d}(u, v)-W_{c}(u, v)=\left(\lambda_{1}-\lambda_{0}\right) \int_{c}^{d} u(t) v(t) d t$, $c, d \in I$, and hence the result is immediate (take limits if $c=a$ or $d=b$ ).

Lemma 2.2. Let $\lambda \in \mathbb{R} \backslash \sigma_{\text {ess }}(H)$. Then

$$
W_{x}\left(u_{ \pm}(\lambda), \dot{u}_{ \pm}(\lambda)\right)=\left\{\begin{array}{l}
-\int_{x}^{b} u_{+}(\lambda, t)^{2} d t \\
\int_{a}^{x} u_{-}(\lambda, t)^{2} d t
\end{array}\right.
$$

where the dot denotes a derivative with respect to $\lambda$.

Proof. From Lemma 2.1 we know that

$$
W_{x}\left(u_{ \pm}(\lambda), u_{ \pm}(\tilde{\lambda})\right)=(\tilde{\lambda}-\lambda)\left\{\begin{array}{l}
-\int_{x}^{b} u_{+}(\lambda, t) u_{+}(\tilde{\lambda}, t) d t \\
\int_{a}^{x} u_{-}(\lambda, t) u_{-}(\tilde{\lambda}, t) d t
\end{array}\right.
$$

Now use this to evaluate the $\operatorname{limit}_{\lim _{\tilde{\lambda} \rightarrow \lambda}} W_{x}\left(u_{ \pm}(\lambda),\left(u_{ \pm}(\lambda)-u_{ \pm}(\tilde{\lambda})\right) /(\lambda-\tilde{\lambda})\right)$.

\section{Oscillation THEORY}

We first introduce Prüfer variables for $u \in C(I, \mathbb{R})$ defined by

$$
u_{1}(x)=\rho_{u}(x) \sin \left(\theta_{u}(x)\right) \quad u_{2}(x)=\rho_{u}(x) \cos \left(\theta_{u}(x)\right) .
$$

If $u$ is never $(0,0)$ and $u$ is continuous, then $\rho_{u}$ is positive and $\theta_{u}$ is uniquely determined once a value of $\theta_{u}\left(x_{0}\right), x_{0} \in I$, is chosen by the requirement $\theta_{u} \in$ $C(I, \mathbb{R})$.

Clearly

$$
W_{x}(u, v)=\rho_{u}(x) \rho_{v}(x) \sin \left(\theta_{u}(x)-\theta_{v}(x)\right) .
$$

An important role is played by the following observation.

Lemma 3.1. Let $\lambda_{0}<\lambda_{1}$, let $u, v$ solve $\tau u=\lambda_{0} u, \tau v=\lambda_{1} v$, and introduce

$$
\Delta_{u, v}(x)=\theta_{u}(x)-\theta_{v}(x) .
$$

Then, if $\Delta_{u, v}(x) \equiv 0 \bmod \pi$,

$$
\lim _{x \rightarrow x_{0}} \frac{\Delta_{u, v}(x)-\Delta_{u, v}\left(x_{0}\right)}{x-x_{0}}=\left(\lambda_{1}-\lambda_{0}\right)>0 .
$$

Proof. If $\Delta_{u, v}\left(x_{0}\right) \equiv 0 \bmod \pi$, then (from (3.2))

$$
\lim _{x \rightarrow x_{0}} \frac{\rho_{u}(x) \rho_{v}(x) \sin \left(\Delta_{u, v}(x)\right)}{x-x_{0}}=W_{x_{0}}^{\prime}(u, v)>0
$$

implies the assertion using (2.1).

Or, put differently, the last proposition implies that the integer part of $\Delta_{u, v}(x) / \pi$ is increasing. 
Lemma 3.2. Let $\lambda_{0}<\lambda_{1}$ and let $u, v$ solve $\tau u=\lambda_{0} u$, $\tau v=\lambda_{1} v$. Denote by $\#(u, v)$ the number of zeros of $W(u, v)$ inside the interval $(a, b)$. Then

$$
\#(u, v)=\lim _{x \uparrow b} \llbracket \Delta_{u, v}(x) / \pi \rrbracket-\lim _{x \downarrow a} \llbracket \Delta_{u, v}(x) / \pi \rrbracket,
$$

where $\llbracket x \rrbracket$ denotes the integer part of a real number $x$, that is, $\llbracket x \rrbracket=\sup \{n \in \mathbb{Z} \mid n \leq$ $x\}$.

Proof. We start with an interval $\left[x_{0}, x_{1}\right]$ containing no zeros of $W(u, v)$. Hence $\llbracket \Delta_{u, v}(x) / \pi \rrbracket=\llbracket \Delta_{u, v}(x) / \pi \rrbracket$. Now let $x_{0} \downarrow a, x_{1} \uparrow b$ and use Lemma 3.1.

If $\lambda \in \mathbb{R} \backslash \sigma_{\text {ess }}(H)$ holds, then equation (2.2) clearly implies

$$
\dot{\theta}_{+}(\lambda, x)=\frac{\int_{x}^{b} u_{+}(\lambda, t)^{2} d t}{\rho_{+}(\lambda, x)^{2}}>0, \quad \dot{\theta}_{-}(\lambda, x)=-\frac{\int_{a}^{x} u_{-}(\lambda, t)^{2} d t}{\rho_{-}(\lambda, x)^{2}}<0,
$$

where we have abbreviated $\rho_{ \pm}(\lambda, x)=\rho_{u_{ \pm}(\lambda)}(x)$ and $\theta_{ \pm}(\lambda, x)=\theta_{u_{ \pm}(\lambda)}(x)$.

Remark 3.3. We remark that linking zeros of $u_{j}$ to the rotation number $\theta_{u}$ is not possible since (unlike in the Sturm-Liouville case) the integer part of $\theta_{u}$ does not count zeros of $u_{j}$. Indeed, (assuming $\phi$ continuous for a moment) shows that $u_{1}(x)=0$ implies $\theta_{u}^{\prime}\left(x_{0}\right)=\phi_{22}\left(x_{0}\right)-\lambda_{0}$, which is not necessarily positive. Hence the integer part of $\theta_{u} / \pi$ can increase or decrease (or stay the same) at zeros of $u_{1}$ (cf. the discussion at the end of Section 2 in [24]). In addition, this implies that zeros of $u_{ \pm, 1}(\lambda,$.$) , are not monotone with respect to \lambda$. Hence solutions can pick up or lose zeros as $\lambda$ increases. This, in general, destroys the connection between zeros and number of eigenvalues. Moreover, if $\phi_{22}(x)-\lambda_{0}$ vanishes on a subinterval of $I$, then $u_{1}(x)$ can vanish on the same interval (without $u$ being identically zero).

However, if $\phi_{22}$ is bounded from above (resp. below), we can apply standard oscillation theory for values of $\lambda$ with $\phi_{22}(x)-\lambda<0$ (resp. $\phi_{22}(x)-\lambda>0$ ) for all $x \in I$ (cf. Remark 4.10 (ii)). Similarly for $u_{2}$.

To further illustrate these problems we consider the following example, with

$$
\phi=\left(\begin{array}{cc}
\theta^{\prime} & 0 \\
0 & \theta^{\prime}
\end{array}\right) .
$$

We will normalize $\theta\left(x_{0}\right)=0$ for some $x_{0} \in I$. The solution $u$ of $\tau u=\lambda_{0} u$ satisfying the initial condition $u\left(x_{0}\right)=\rho_{0}\left(\sin \theta_{0}, \cos \theta_{0}\right)$ is given by

$$
u(x)=\rho_{0}\left(\begin{array}{c}
\sin \left(\theta_{0}-\lambda_{0} x+\theta(x)\right) \\
\cos \left(\theta_{0}-\lambda_{0} x+\theta(x)\right)
\end{array}\right) .
$$

Clearly, if $\theta^{\prime}(x)=\lambda_{0}$ for $x \in\left(x_{0}, x_{0}+\varepsilon\right)$ and $\theta_{0}=0$, then $u(x)=\left(0, \rho_{0}\right)$ for $x \in\left(x_{0}, x_{0}+\varepsilon\right)$.

To get more specific, let $I=(0,1), \theta(x)=4 x(x-1), x_{0}=0$, and impose the boundary conditions $f_{1}(0)=f_{1}(1)=0$. We easily obtain $\sigma(H)=\pi \mathbb{Z}$ and

$$
\theta_{-}(\lambda, x)=\theta(x)-\lambda x, \quad \theta_{+}(\lambda, x)=\theta(x)-\lambda(x-1) .
$$

This implies the following for the zeros of $u_{-, 1}(\lambda,$.$) as \lambda$ increases. At $\lambda=0 \in \sigma(H)$ there are no zeros. At $\lambda=4(\sqrt{\pi}-1) \notin \sigma(H)$ we pick up two zeros, one of which gets lost again at $\lambda=\pi \in \sigma(H)$. As soon as $\lambda>4$ we have $\theta^{\prime}(x)-\lambda>0$ for all $x \in I$, and from now on $u_{-, 1}(\lambda,$.$) picks up precisely one zero whenever \lambda$ hits an eigenvalue (and no zeros get lost).

To end this remark we compute $\Delta_{u_{-}\left(\lambda_{0}\right), u_{+}\left(\lambda_{1}\right)}(x)=\lambda_{1}(x-1)-\lambda_{0} x$, where all unpleasant factors cancel. 


\section{Number of Eigenvalues AND ZEROS OF Wronskians}

The objective of this section is to establish the connection between zeros of the Wronskian and spectra of Dirac operators. As a warmup we considers the regular case.

Theorem 4.1. Suppose $\tau$ is regular. Denote by $P_{\Omega}(H)$ the family of spectral projections for $H$. Then for $\lambda_{0}<\lambda_{1}$ we have

$$
\operatorname{dim} \operatorname{Ran} P_{\left(\lambda_{0}, \lambda_{1}\right)}(H)=\#\left(u_{-}\left(\lambda_{0}\right), u_{+}\left(\lambda_{1}\right)\right)=\#\left(u_{+}\left(\lambda_{0}\right), u_{-}\left(\lambda_{1}\right)\right),
$$

where $\#(u, v)$ is the number of zeros of $W(u, v)$ inside $(a, b)$.

Proof. We only carry out the proof for the $\#\left(u_{-}\left(\lambda_{0}\right), u_{+}\left(\lambda_{1}\right)\right)$ case. Defining $\#\left(u_{-}\left(\lambda_{0}\right), u_{+}\left(\lambda_{1}\right)\right)$ as in (3.6) shows that our claim is true for $\lambda_{1}$ close to $\lambda_{0}$. Abbreviate $\Delta(\lambda, x)=\Delta_{u_{-}\left(\lambda_{0}\right), u_{+}(\lambda)}(x)$. Since $\Delta(\lambda, b)$ is independent of $\lambda$, it suffices to look at $\Delta(\lambda, a)$. As $\lambda$ increases from $\lambda_{0}$ to $\lambda_{1},-\Delta(\lambda, a)$ increases by (3.7) and is $0 \bmod \pi$ if and only if $\lambda$ is an eigenvalue of $H$ (Lemma 3.2, equation (1.6)), completing the proof.

Next, we want to prove Theorem 4.1 in the general case. This will be done in two parts.

Theorem 4.2. Let $\lambda_{0}<\lambda_{1}$ and $\sigma_{0}, \sigma_{1} \in\{ \pm\}$. Suppose $u_{\sigma_{j}}\left(\lambda_{j},.\right), j=0,1$, exist. Then

$$
\operatorname{dim} \operatorname{Ran} P_{\left(\lambda_{0}, \lambda_{1}\right)}(H) \geq \#\left(u_{\sigma_{0}}\left(\lambda_{0}\right), u_{\sigma_{1}}\left(\lambda_{1}\right)\right)
$$

Proof. Again the proof is only done for $\sigma_{0}=-$. Abbreviate $u=u_{-}\left(\lambda_{0}\right)$ and $v=$ $u_{+}\left(\lambda_{1}\right)$ and $n=\#(u, v)$. Suppose $n$ finite; otherwise the following argument works for arbitrary large $n$. Let $x_{1}, \ldots, x_{n}$ be the zeros of $W_{x}(u, v)$. Since $W_{x_{j}}(u, v)=0$, there exists constants $\gamma_{j}$ such that

$$
\eta_{j}(x)=\left\{\begin{array}{cl}
u(x), & x \leq x_{j}, \quad 1 \leq j \leq n, \\
\gamma_{j} v(x), & x>x_{j},
\end{array}\right.
$$

is in the domain of $H$ (i.e., $\left.u\left(x_{j}\right)=\gamma_{j} v\left(x_{j}\right)\right)$. Furthermore, set

$$
\tilde{\eta}_{j}(x)= \begin{cases}-u(x), & x \leq x_{j}, \quad 1 \leq j \leq n . \\ \gamma_{j} v(x), & x>x_{j},\end{cases}
$$

If $\lambda_{1}$ is an eigenvalue of $H$ we define in addition $\eta_{0}=v=\tilde{\eta}_{0}, x_{0}=a$, and if $\lambda_{0}$ is an eigenvalue of $H, \eta_{n+1}=u=-\tilde{\eta}_{n+1}, x_{n+1}=b$. Lemma 2.1 implies $\int_{x_{j}}^{x_{k}} u v d x=0$, and hence $\int_{b}^{a} \eta_{j} \eta_{k} d x=\int_{b}^{a} \tilde{\eta}_{j} \tilde{\eta}_{k} d x$ for all $j, k$. Using

$$
\left(H-\frac{\lambda_{1}+\lambda_{0}}{2}\right) \eta_{j}=\frac{\lambda_{1}-\lambda_{0}}{2} \tilde{\eta}_{j},
$$

we obtain

$$
\left\|\left(H-\frac{\lambda_{1}+\lambda_{0}}{2}\right) \eta\right\|=\frac{\lambda_{1}-\lambda_{0}}{2}\|\eta\|
$$

for any $\eta$ in the span of the $\eta_{j}$ 's. Thus, $\operatorname{dim} \operatorname{Ran} P_{\left[\lambda_{0}, \lambda_{1}\right]}(H) \geq \operatorname{dim}\left(\operatorname{span}\left\{\eta_{j}\right\}\right)$. But $u$ and $v$ are independent on each interval (since their Wronskian is non-constant), and so the $\eta_{j}$ are linearly independent. This proves the theorem in the $u=u_{-}\left(\lambda_{0}\right)$, $v=u_{+}\left(\lambda_{1}\right)$ case. 
The case $u=u_{-}\left(\lambda_{0}\right), v=u_{-}\left(\lambda_{1}\right)$ is similar. We define

$$
\eta_{j}(x)=\left\{\begin{array}{cl}
u(x)+\gamma_{j} v(x), & x \leq x_{j}, \quad 1 \leq j \leq n \\
0, & x>x_{j},
\end{array}\right.
$$

(with $\eta_{j} \in \mathfrak{D}(H)$ ), and

$$
\tilde{\eta}_{j}(x)=\left\{\begin{array}{cl}
-u(x)+\gamma_{j} v(x), & x \leq x_{j}, \quad 1 \leq j \leq n . \\
0, & x>x_{j},
\end{array}\right.
$$

If $\lambda_{1}$ is an eigenvalue of $H$ we define in addition $\eta_{0}=v=\tilde{\eta}_{0}, x_{0}=b$, and if $\lambda_{0}$ is an eigenvalue of $H, \eta_{n+1}=u=-\tilde{\eta}_{n+1}, x_{n+1}=b$. Again, the $\eta_{j}$ 's are linearly independent by considering their supports. And since $\int_{a}^{x_{j}} u v d x=0,1 \leq j \leq n$, we can proceed as before.

Fix functions $u, v$. Pick $a_{m} \downarrow a, b_{m} \uparrow b$ and set $I_{m}=\left(a_{m}, b_{m}\right)$. Define $\tilde{H}_{m}$ : $\mathfrak{D}\left(\tilde{H}_{m}\right) \rightarrow L^{2}\left(I_{m}, \mathbb{C}^{2}\right), f \mapsto \tau f$, with

$$
\begin{aligned}
& \mathfrak{D}\left(\tilde{H}_{m}\right)=\left\{f \in L^{2}\left(I_{m}, \mathbb{C}^{2}\right) \mid f \in A C\left(I_{m}, \mathbb{C}^{2}\right), \tau f \in L^{2}\left(I_{m}, \mathbb{C}^{2}\right),\right. \\
& \left.W_{a_{m}}(u, f)=W_{b_{m}}(v, f)=0\right\} \text {. }
\end{aligned}
$$

Consider $H_{m}=\alpha \mathbb{1} \oplus \tilde{H}_{m} \oplus \alpha \mathbb{1}$ on $L^{2}\left(I, \mathbb{C}^{2}\right)=L^{2}\left(\left(a, a_{m}\right), \mathbb{C}^{2}\right) \oplus L^{2}\left(I_{m}, \mathbb{C}^{2}\right) \oplus$ $L^{2}\left(\left(b_{m}, b\right), \mathbb{C}^{2}\right)$, where $\alpha$ is a fixed real constant. Then we have the following standard result ([22], Chapter 16, [24], Section 1, and [4], Section 5).

Lemma 4.3. Suppose that either $H$ is limit point at a or that $u=u_{-}\left(\lambda_{0}\right)$ for some $\lambda_{0}$ and similarly, that either $H$ is limit point at $b$ or $v=u_{+}\left(\lambda_{1}\right)$ for some $\lambda_{1}$. Then $H_{m}$ converges to $H$ in the strong resolvent sense as $m \rightarrow \infty$, and hence

$$
\operatorname{dim} \operatorname{Ran} P_{\left(\lambda_{0}, \lambda_{1}\right)}(H) \leq \lim \inf \operatorname{dim} \operatorname{Ran} P_{\left(\lambda_{0}, \lambda_{1}\right)}\left(H_{m}\right) .
$$

Now we are ready to prove

Theorem 4.4. If $u=u_{\mp}\left(\lambda_{0}\right)$ and $v=u_{ \pm}\left(\lambda_{1}\right)$, then

$$
\operatorname{dim} \operatorname{Ran} P_{\left(\lambda_{0}, \lambda_{1}\right)}(H) \leq \#(u, v) .
$$

If $H$ is limit point at $b$ (resp. a), we can replace $u_{-}\left(\lambda_{j}\right)$ (resp. $u_{+}\left(\lambda_{j}\right)$ ) by an arbitrary solution of $\tau u=\lambda_{j} u$.

Proof. We can assume $\#(u, v)<\infty$ (otherwise there is nothing to prove). Pick $a_{m} \downarrow a, b_{m} \uparrow b$. Let $H_{m}$ be given as in Lemma 4.3 with $\alpha \notin\left[\lambda_{0}, \lambda_{1}\right]$. If $m$ is so large that all zeros of $W(u, v)$ are in $\left(a_{m}, b_{m}\right)$, Theorem 4.1 implies $\#(u, v)=$ $\operatorname{dim} \operatorname{Ran} P_{\left(\lambda_{0}, \lambda_{1}\right)}\left(\tilde{H}_{m}\right)=\operatorname{dim} \operatorname{Ran} P_{\left(\lambda_{0}, \lambda_{1}\right)}\left(H_{m}\right)$, since $\alpha \notin\left[\lambda_{0}, \lambda_{1}\right]$. Thus, by Lemma 4.3 , (4.11) holds, as was to be proven.

Combining the last two theorems, we get

Theorem 4.5. Let $\lambda_{0}<\lambda_{1}$. Then

$$
\operatorname{dim} \operatorname{Ran} P_{\left(\lambda_{0}, \lambda_{1}\right)}(H)=\#\left(u_{-}\left(\lambda_{0}\right), u_{+}\left(\lambda_{1}\right)\right)=\#\left(u_{+}\left(\lambda_{0}\right), u_{-}\left(\lambda_{1}\right)\right),
$$

where \# $(u, v)$ denotes the number of zeros of $W(u, v)$ inside $(a, b)$. The result still holds for $u=u_{-}\left(\lambda_{0}\right), v=u_{-}\left(\lambda_{1}\right)$ (resp. $u=u_{+}\left(\lambda_{0}\right), v=u_{+}\left(\lambda_{1}\right)$ ) if $H$ is limit point at $b$ (resp. a).

Remark 4.6. The limit point assumption in the case $u=u_{\mp}\left(\lambda_{0}\right), v=u_{\mp}\left(\lambda_{1}\right)$ is clearly crucial, since the Wronskian contains no information about the boundary condition at $a$ respectively $b$ in this case. 
Finally, we state

Theorem 4.7. Let $\lambda_{0} \neq \lambda_{1}$. Let $\tau u=\lambda_{0} u, \tau v=\lambda_{1} v$, and $\tau \tilde{v}=\lambda_{1} \tilde{v}$ with $v$ independent of $\tilde{v}$. Then the zeros of $W(u, v)$ interlace the zeros of $W(u, \tilde{v})$ (in the sense that there is exactly one zero of one function between two adjacent zeros of the other $)$. In particular, $|\#(u, v)-\#(u, \tilde{v})| \leq 1$.

Proof. The result is immediate from $0<\Delta_{v, \tilde{v}}(x)<\pi$ (for a suitable normalization of $\left.\Delta_{v, \tilde{v}}(x)\right)$, which follows from the constancy of $W(v, \tilde{v})$.

By applying this theorem twice, we deduce

Theorem 4.8. Let $\lambda_{0} \neq \lambda_{1}$. Let $u, \tilde{u}$ and $v, \tilde{v}$ be the linearly independent solutions of $\tau u=\lambda_{0} u$ and $\tau v=\lambda_{1} v$, respectively. Then

$$
|W(u, v)-W(\tilde{u}, \tilde{v})| \leq 2 .
$$

Moreover, we infer the following useful result.

Corollary 4.9. Let $u, v$ satisfy $\tau u=\lambda_{0} u, \tau v=\lambda_{1} v$. Then

$$
\#(u, v)<\infty \quad \Leftrightarrow \quad \operatorname{dim} \operatorname{Ran} P_{\left(\lambda_{0}, \lambda_{1}\right)}(H)<\infty
$$

Proof. Using the split-up $H_{x_{0},-} \oplus H_{x_{0},+}$ reduces the problems to the case with one regular endpoint. Thus the solutions $u_{ \pm}(\lambda)$ exist at at least one end point. Using first Theorem 4.5 and then Theorem 4.8 finishes the proof.

Remark 4.10. (i). We remark that all results obtained thus far also hold for the more general system

$$
\tau=k(x)^{-1}\left(\frac{1}{\mathrm{i}} \sigma_{2}\left(p(x) \frac{d}{d x}+\frac{d}{d x} p(x)\right)+\phi(x)\right),
$$

where $p \in A C_{l o c}(I,(0, \infty))$ and $k$ is a symmetric positive definite matrix with coefficients $k_{i j} \in L_{l o c}^{1}(I, \mathbb{R})$. The necessary modifications are straightforward (see also [24], Section 5).

(ii). In the case of supersymmetric Dirac operators (i.e., $\phi_{11}=\phi_{22}=0$ )

$$
H=\left(\begin{array}{cc}
0 & A^{*} \\
A & 0
\end{array}\right), \quad A=\frac{d}{d x}+\phi_{12}(x)
$$

(note that $H$ and $-H$ are unitarily equivalent) we have

$$
H^{2}=\left(\begin{array}{cc}
H_{1} & 0 \\
0 & H_{2}
\end{array}\right), \quad H_{1}=A A^{*}, H_{2}=A^{*} A .
$$

Moreover, $\tau u=\lambda u$ implies $\tau_{j} u_{j}=-u_{j}^{\prime \prime}+\left(\phi_{12}^{2}-(-1)^{j} \phi_{12}^{\prime}\right) u_{j}=\lambda u_{j}, j=1,2$, where $\tau_{j}$ is the differential expression corresponding to $H_{j}$. This says that all oscillation theoretic results for supersymmetric Dirac operators follow immediately from the corresponding results for (semi-bounded) one-dimensional Schrödinger operators.

\section{Applications}

In our final section we want to apply our results to investigate the spectra of short-range perturbations of periodic Dirac operators. Our objective is to prove the analog of the theorem of Rofe-Beketov [15] about the finiteness of the number of eigenvalues in essential spectral gaps of the perturbed Hill operator. The reader 
might find some results for the special case of perturbed constant operators in [10], [3] and for the general case in [8], [9].

We first recall some basic facts from the theory of periodic Dirac operators (cf., e.g., [1], [21], [22], Chapter 12). Let $H_{p}$ be a Dirac operator associated with the periodic potential $\phi_{p}$, that is, $\phi_{p}(x+1)=\phi_{p}(x), x \in I=\mathbb{R}$. The spectrum of $H_{p}$ is purely absolutely continuous and consists of a countable number of gaps, that is,

$$
\sigma\left(H_{p}\right)=\bigcup_{j \in \mathbb{Z}}\left[E_{2 j}, E_{2 j+1}\right]
$$

with $\cdots<E_{2 j}<E_{2 j+1} \leq E_{2 g+2}<E_{2 g+3}<\cdots$. Moreover, Floquet theory implies the existence of solutions $u_{p, \pm}(z,$.$) of \tau_{p} u=z u, z \in \mathbb{C}\left(\tau_{p}\right.$ the differential expression corresponding to $H_{p}$ ), satisfying

$$
u_{p, \pm}(z, x)=p_{ \pm}(z, x) m(z)^{ \pm x}, \quad p_{ \pm}(z, x+1)=p_{ \pm}(z, x),
$$

where $m(z) \in \mathbb{C}$ is called the Floquet multiplier. $m(z)$ satisfies $m(z)^{2}=1$ for $z \in\left\{E_{j}\right\}_{j=0}^{2 g+1},|m(z)|=1$ for $z \in \sigma\left(H_{p}\right)$, and $|m(z)|<1$ for $z \in \mathbb{C} \backslash \sigma\left(H_{p}\right)$. (This says, in particular, that $u_{p, \pm}(z,$.$) are bounded for z \in \sigma\left(H_{p}\right)$ and linearly independent for $z \in \mathbb{C} \backslash\left\{E_{j}\right\}_{j \in \mathbb{Z}}$.)

As anticipated, we will study perturbations $H$ of $H_{p}$ associated with a potential satisfying $\phi(x) \rightarrow \phi_{p}(n)$ as $|x| \rightarrow \infty$. Both $H$ and $H_{p}$ are limit point (cf. [25], Satz 5.1) and hence give rise to a unique self-adjoint operator when defined maximally. Using this notation, our theorem reads:

Theorem 5.1. Suppose $\phi_{p}$ is a given periodic potential and $H_{p}$ is the corresponding Dirac operator. Let $H$ be a perturbation of $H_{p}$ such that

$$
\int_{\mathbb{R}}(1+|x|)\left|\phi(x)-\phi_{p}(x)\right| d x<\infty .
$$

Then $\sigma_{\text {ess }}(H)=\sigma\left(H_{p}\right)$, and the point spectrum of $H$ is confined to the spectral gaps of $H_{p}$, that is, $\sigma_{p}(H) \subset \mathbb{R} \backslash \sigma\left(H_{p}\right)$, and finite in each gap. Furthermore, the essential spectrum of $H_{p}$ is purely absolutely continuous.

Proof. Using (1.8) plus $\left|u_{p, \pm}(z, x)\right| \leq C_{ \pm}|m(z)|^{ \pm x}$ shows that $H$ is relatively compact with respect to $H_{p}$, implying $\sigma_{e s s}(H)=\sigma_{e s s}\left(H_{p}\right)$. To prove the remaining claims it suffices to show the existence of solutions $u_{ \pm}(\lambda,$.$) of \tau u=\lambda u$ for $\lambda \in \sigma\left(H_{p}\right)$ (continuous w.r.t. $\lambda$ ) satisfying

$$
\lim _{n \rightarrow \pm \infty}\left|u_{ \pm}(\lambda, x)-u_{p, \pm}(\lambda, x)\right|=0 .
$$

In fact, for $\lambda \in \sigma\left(H_{p}\right)$ there exists at least one bounded solution which is not square integrable, and hence there are no eigenvalues in the essential spectrum of $H$ (since the Wronskian of a bounded and a square integrable solution must vanish). Invoking Theorem XIII.20 of [13] shows that the essential spectrum of $H$ is purely absolutely continuous. Moreover, since $W_{x}\left(u_{p,-}\left(E_{2 j-1}\right), u_{p,+}\left(E_{2 j}\right)\right)$ has no zeros, we infer that $W_{x}\left(u_{-}\left(E_{2 j-1}\right), u_{+}\left(E_{2 j}\right)\right)$ has only finitely many zeros. Thus by Corollary 4.9 there are only finitely many eigenvalues in each gap. It remains to show (5.4). Suppose $u_{+}(\lambda,),. \lambda \in \sigma\left(H_{p}\right)$, satisfies

$$
u_{ \pm}(\lambda, x)=u_{p, \pm}(\lambda, x)-\mathrm{i} \sigma_{2} \int_{ \pm \infty}^{x} U_{p}(\lambda, x, y)\left(\phi(y)-\phi_{p}(y)\right) u_{ \pm}(\lambda, y) d y
$$


where $U_{p}(\lambda, ., y)$ is the fundamental matrix solution of of $\tau_{p} u=\lambda u$ satisfying the initial conditions $U_{p}(\lambda, y, y)=\mathbb{1}$. Then $u_{ \pm}(\lambda,$.$) satisfies \tau u=\lambda u$ and (5.4). Existence of a solution of (5.5) follows upon applying a standard iteration argument (compare also [8] and [20] in the special case $\phi_{p}=0$ ) using

$$
\left|U_{p}(\lambda, x, y)\right| \leq C(1+|x-y|), \quad \lambda \in \sigma\left(H_{p}\right), C>0 .
$$

Clearly, there are several other strategies to prove Theorem 5.1. The proof given here has the advantage of being rather short and transparent. In addition, the idea of proof applies to more general scattering situations (where $H_{p}$ is not necessarily periodic) as long as sufficient information about the spectrum of $H_{p}$ and the asymptotic behavior of (weak) solutions of $H_{p}$ and $H$ is available.

Remark 5.2. The fact that the essential spectrum of $H$ is purely absolutely continuous was first proven by [9] under the weaker assumption $\int_{\mathbb{R}}\left|\phi(x)-\phi_{p}(x)\right| d x<\infty$. Since (5.3) is only needed to ensure existence of $u_{ \pm}(\lambda, x)$ for $\lambda$ at the boundary of $\sigma\left(H_{p}\right)$ (for $\lambda$ in the interior of $\sigma\left(H_{p}\right)$ we have $\left|U_{p}(\lambda, x, y)\right| \leq C$ ) the weaker assumption above suffices), our proof also covers this situation. However, the example

$$
\phi(x)=\phi_{p}(x)+\left(\begin{array}{cc}
\frac{x^{2}-1}{\left(x^{2}+1\right)^{2}} & 0 \\
0 & 0
\end{array}\right), \quad \phi_{p}(x)=\left(\begin{array}{cc}
1 & 0 \\
0 & -1
\end{array}\right)
$$

shows that (5.3) cannot be replace by $\int_{\mathbb{R}}\left(1+|x|^{\varepsilon}\right)\left|\phi(x)-\phi_{p}(x)\right| d x<\infty, \varepsilon<1$. Indeed, $H$ has an eigenvalue $1 \in \sigma\left(H_{p}\right)=(-\infty,-1] \cup[1, \infty)$ with corresponding eigenfunction

$$
u(1, x)=\frac{1}{\left(x^{2}+1\right)^{2}}\left(\begin{array}{c}
x^{2}+1 \\
-x
\end{array}\right)
$$

\section{ACKNowledgments}

I thank S. Timischl for discussions, and F. Gesztesy, B. Thaller, and K. Unterkofler for hints with respect to the literature.

\section{REFERENCES}

[1] W. Bulla, F. Gesztesy, and K. Unterkofler On relativistic energy band corrections in the presence of periodic potentials, Lett. Math. Phys. 15, 313-324 (1988). MR 90c:81038

[2] W.A. Coppel Disconjugacy, Lecture Notes in Mathematics 220, Springer, Berlin 1971. MR $\mathbf{5 7 : 7 7 8}$

[3] I.S. Frolov, Inverse scattering problem for a Dirac system on the whole axis, Soviet Math. Dokl. 13, 1468-1472 (1972). MR 47:5352

[4] F. Gesztesy, B. Simon, and G. Teschl, Zeros of the Wronskian and renormalized oscillation theorems, Am. J. Math. 118, 571-594 (1996). CMP 96:13

[5] P. Hartman, Differential equations with non-oscillatory eigenfunctions, Duke Math. J. 15, 697-709 (1948). MR 10:376e

[6] P. Hartman, A characterization of the spectra of one-dimensional wave equations, Am. J. Math. 71, 915-920 (1949). MR 11:438a

[7] P. Hartman and C.R. Putnam, The least cluster point of the spectrum of boundary value problems, Am. J. Math. 70, 849-855 (1948). MR 10:376f

[8] D.B. Hinton and C.K. Shaw, Asymptotics of solutions and spectra of perturbed periodic Hamiltonian systems, in "Differential Equations and Mathematical Physics", (I.W. Knowles and Y. Saito Eds.), 169-174, Lecture Notes in Mathematics 1285, Springer, Berlin 1987. MR 89g:34026 
[9] D.B. Hinton and C.K. Shaw, Absolutely continuous spectra of perturbed periodic Hamiltonian systems, Rocky Mtn. J. Math., 727-748 (1987). MR 89m:34031

[10] D.B. Hinton, A.B. Mingarelli, T.T. Read, and C.K. Shaw, On the number of eigenvalues in the spectral gap of a Dirac system, Proc. of the Edinburgh Math. Soc. 29, 367-378 (1986). MR 88d:34029

[11] K. Kreith Oscillation Theory, Lecture Notes in Mathematics 324, Springer, Berlin 1973.

[12] B.M. Levitan and I.S. Sargsjan, Sturm-Liouville and Dirac Operators, Kluwer Academic Publishers, Dordrecht 1991. MR 92i:34119

[13] M. Reed and B. Simon, Methods of Modern Mathematical Physics IV. Analysis of Operators, Academic Press, San Diego, 1978. MR 58:12429c

[14] W.T. Reid Sturmian Theory for Ordinary Differential Equations, Springer, New York 1980. MR 82f:34002

[15] F.S. Rofe-Beketov, A test for the finiteness of the number of discrete levels introduced into gaps of a continuous spectrum by perturbations of a periodic potential, Soviet Math. Dokl. 5, 689-692 (1964). MR 28:4176

[16] J.C.F. Sturm Mémoire sur les équations différentielles linéaires du second ordre, J. Math. Pures Appl. 1, 106-186 (1836).

[17] C.A. Swanson Comparison and Oscillation Theory of Linear Differential Equations, Academic Press, New York 1968. MR 57:3515

[18] G. Teschl, Oscillation theory and renormalized oscillation theory for Jacobi operators, J. Diff. Eqs. 129, 532-558 (1996). CMP 96:17

[19] B. Thaller, The Dirac Equation, Springer, Berlin, 1992. MR 94k:81056

[20] S. Timischl, A trace formula for one-dimensional Dirac operators, diploma thesis, University of Graz, Austria, 1995.

[21] K. Unterkofler, Periodische Potentiale in der eindimensionalen Diracgleichung, diploma thesis, Technical University of Graz, Austria, 1986.

[22] J. Weidmann, Spectral Theory of Ordinary Differential Operators, Lecture Notes in Mathematics 1258, Springer, Berlin 1987. MR 89b: 47070

[23] J. Weidmann, Zur Spektraltheorie von Sturm-Liouville Operatoren, Math. Z. 98, 268-302 (1971). MR 35:4769

[24] J. Weidmann, Ozillationsmethoden für Systeme gewönlicher Differentialgleichungen, Math. Z. 119, 349-337 (1971). MR 44:2975

[25] J. Weidmann, Absolut stetiges Spektrum bei Sturm-Liouville und Dirac-Systemen, Math. Z. 180, 423-427 (1982). MR 83m:34023

Institut für Reine und Angewandte Mathematik, RWTH Aachen, 52056 Aachen, GerMANY

Current address: Institut für Mathematik, Universität Wien, Strudelhofgasse 4, 1090 Vienna, Austria

E-mail address: gerald@mat.univie.ac.at

URL: http://www.mat.univie.ac.at/〜gerald/ 